Article

\title{
Polymer M-Z Thermal Optical Switch at 532-nm Based on Wet Etching and UV-Writing Waveguide
}

\author{
Jiawen Lv, Yue Cao, Baizhu Lin, Yue Yang, Yue Sun, Shuai Li, Yunji Yi * $\mathbb{D}$, Fei Wang \\ and Daming Zhang
}

State Key Laboratory of Integrated Optoelectronics, College of Electronic Science \& Engineering, Jilin University, Changchun 130012, China; lvjw18@mails.jlu.edu.cn (J.L.); yuecao17@mail.jlu.edu.cn (Y.C.); linbz17@mails.jlu.edu.cn (B.L.); a2604702999@163.com (Y.Y.); sun16@mails.jlu.edu.cn (Y.S.); 17607178920@163.com (S.L.); wang_fei@jlu.edu.cn (F.W.); zhangdm@jlu.edu.cn (D.Z.)

* Correspondence: yiyj@jlu.edu.cn; Tel.: +86-0431-8516-8097

Received: 28 February 2019; Accepted: 25 May 2019; Published: 4 June 2019

\begin{abstract}
Polymer thermal optical switches have low power consumption and $532 \mathrm{~nm}$ is the communication window of polymer fiber. Polymer thermal optical switches at $532 \mathrm{~nm}$ are rarely reported, because of switching extinction ratio properties that are restricted by modes of the waveguide. Single mode waveguide at $532 \mathrm{~nm}$ is hard to fabricate due to the dissolution of core and cladding materials. A polymer M-Z thermal optical switch at $532 \mathrm{~nm}$ was first demonstrated based on the wet etching method. The proposed thermal optical switch was consisted of silica substrate, photosensitive polymer core, and cladding material. The device was fabricated and tested with the power consumption of $6.55 \mathrm{~mW}$, extinction of $4.8 \mathrm{~dB}$, and switching time of $0.23 \mathrm{~ms}$ (rise) $/ 0.28 \mathrm{~ms}$ (down). An optimized switch structure combining with the UV-writing technique and graphene thermal conduction layer was proposed based on the experiments above. A side electrode was designed to reduce the power consumption and the switching time. The optimized device was calculated to have a power consumption of $1.5 \mathrm{~mW}$. The switching time of the UV-writing device was simulated to be $18.2 \mu \mathrm{s}$ (rise) and $85 \mu \mathrm{s}$ (down). The device is promising in the wearable device and laser radar area.
\end{abstract}

Keywords: polymer waveguide; thermal optical effect; UV-writing; optical switch

\section{Introduction}

As a new method of communication, optical signal achieves faster network speed and wider bandwidth. Optical switch is an important device for optoelectronic and integrated optics. It plays the role of protecting, testing, monitoring, and management of the network. The thermal optical (TO) switch, as the important part of the optical switch, acts a pivotal part in showing great promise in low-speed fiber-optic communication systems [1-3]. At present, the wavelengths of the reported thermal optical switches are mostly at $1550 \mathrm{~nm}$ and $650 \mathrm{~nm}$, and the thermal optical switch working at $650 \mathrm{~nm}$ is mainly used in short-range communication system [4-6]. In recent years, $532 \mathrm{~nm}$ wavelength has gained wide attention in areas of industrial control, photobiology, and short-range communication. Corresponding lasers and detectors have been widely used. The corresponding optical signal processing devices art the chip level are progressing. In particular, optical switches in the photonic integrated circuit at $532 \mathrm{~nm}$ wavelength are promising in optical computing chip, wearable photobiology chip, and underwater laser radar [7,8].

Polymer materials have a high thermal optical coefficient, which can significantly reduce power consumption of thermal optical devices. At present, the polymer waveguides mainly include all-polymer devices and hybrid polymer/silica devices. In 2006, Young-Ouk Noh reported a thermal 
optical of polymer waveguide, and the switching crosstalk is reduced to $-70 \mathrm{~dB}$ with an applied electrical power of $200 \mathrm{~mW}$ [9]. In 2013, Changming Chen reported an all-polymer thermal optical waveguide switch arrays based on novel grafting poly (methyl methacrylate) (PMMA) materials. The $\mathrm{M}-\mathrm{Z}$ interferometer thermal optical switch on-off time is $600 \mu \mathrm{s}$. The extinction ratio is about $8.2 \mathrm{~dB}$ and the applied electric power as the switching power is about $22.6 \mathrm{~mW}$ at $650 \mathrm{~nm}$. The all-polymer thermal optical switch has the advantage of low power consumption, but the response time is longer [10]. In 2012, Lei Liang fabricated a Mach-Zehnder interferometer (MZI) thermal optical switch at $1550 \mathrm{~nm}$ wavelength while using a hybrid silica/polymer waveguide structure. The switching power is $13 \mathrm{~mW}$ and the extinction ratio is $18.3 \mathrm{~dB}$ with the rise/fall time of 73.5/96.5 $\mu \mathrm{s}$, respectively [11]. The hybrid silica/polymer structure ensures low power consumption and reduces the switching time. Therefore, the organic-inorganic hybrid integrated waveguide structure was selected in this paper.

The thermal optical switch can be divided into Mach-Zehnder (M-Z) thermal optical switch, multimode-interference (MMI) thermal optical switch, $\mathrm{X}$-band thermal optical switch, and digital thermal optical switch [12-14]. M-Z optical switch has large process tolerance and lower power consumption. With the $\mathrm{M}-\mathrm{Z}$ structure, the waveguide mode will have an effect on the extinction ratio and other parameters of the device. Therefore, it is crucial to achieve a single mode waveguide. For polymer materials, ridge the waveguide structure is an effective method to achieve single mode waveguide $[15,16]$. However, the single mode ridge polymer waveguide at $532 \mathrm{~nm}$ has large coupling loss between the device and the fiber. Another method is adjusting the refractive index of polymers. Due to the spin coating fabrication process, the waveguides with close refractive index difference are usually suffering from the core-cladding solution problem. Therefore, the high-quality single-mode waveguide is the key factor to the $532 \mathrm{~nm} \mathrm{M-Z} \mathrm{thermal} \mathrm{optical} \mathrm{switch.}$

In this paper, we firstly designed and fabricated a M-Z thermal optical switch at $532 \mathrm{~nm}$ with a top electrode using the wet etching method. We then proposed a UV-writing method [17-19] to achieve few modes waveguide in order to optimize the extinction ratio. The side electrode structure without cladding was introduced to guarantee fast switching properties. The graphene thermal conduction layer would insure the high heating efficiency. The device parameters and performance were analyzed.

\section{Materials and Methods}

\subsection{SU-8/PMMA Wet Etching M-Z Optical Switch with Top Electrode}

Figure 1a shows the device structure. SU-8 is selected as the polymer waveguide core material due to its easy fabrication method. Its refractive index and thermal conductivity are 1.595 and $0.2 \mathrm{~W} /(\mathrm{m} \cdot \mathrm{K})$, respectively. Silicon is selected as the substrate and heat sink with $5 \mu \mathrm{m}$ silica as the lower cladding. The refractive index and thermal conductivity of silica are 1.461 and $1.4 \mathrm{~W} /(\mathrm{m} \cdot \mathrm{K})$, respectively. PMMA is selected as the polymer upper cladding material, and its refractive index and thermal conductivity are 1.494 and $0.19 \mathrm{~W} /(\mathrm{m} \cdot \mathrm{K})$, respectively. The $\mathrm{M}-\mathrm{Z}$ structure parameters are shown in Figure 1a with the branch width of $30 \mu \mathrm{m}$, the branch length of $1 \mathrm{~cm}$, and the $Y$ branch length of $0.1 \mathrm{~cm}$. A single mode waveguide at $532 \mathrm{~nm}$ wavelength needs a waveguide dimension of $0.3 \mu \mathrm{m} \times 0.9 \mu \mathrm{m}$, according to Figure $1 \mathrm{~b}$. This dimension cannot be fabricated by lithography. In addition, it will increase the coupling loss. Therefore, the waveguide dimension is selected to be $3 \mu \mathrm{m} \times 9 \mu \mathrm{m}$.

Comsol Multiphysics and Matlab simulated the optical field and thermal field. Figure $2 a$ is the optical field profile of the fundamental mode, Figure $2 \mathrm{~b}$ shows the thermal field. The rise time and fall time of the device was simulated to be $218.55 \mu$ s and $372.79 \mu$ s, respectively. However, fiber coupling will excite all of the modes at the same time in the waveguide. Subsequently, the device cannot provide a meaningful low crosstalk. 


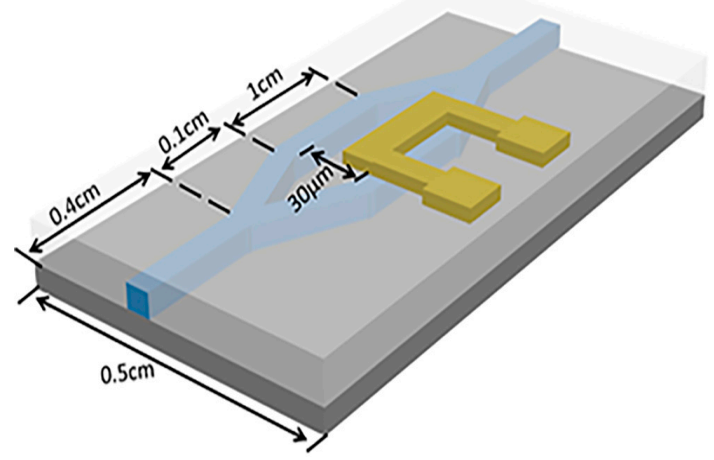

(a)

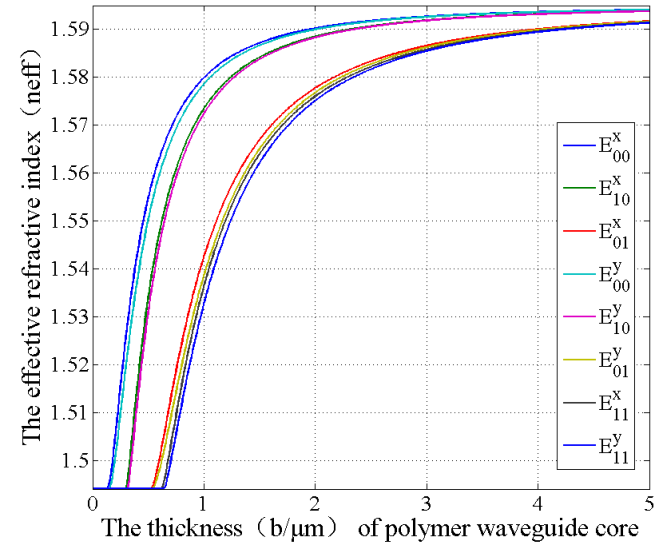

(b)

Figure 1. (a) The schematic of polymer/silica hybrid thermal optical switch. (b) The single mode condition of the polymer waveguide at $532 \mathrm{~nm}$ (The aspect ratio is 0.333 ).

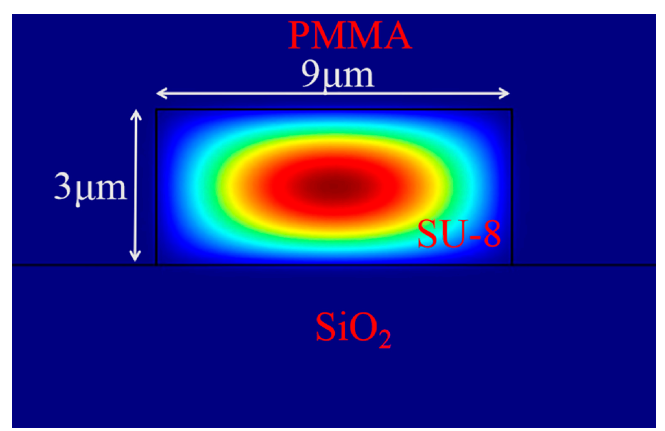

(a)

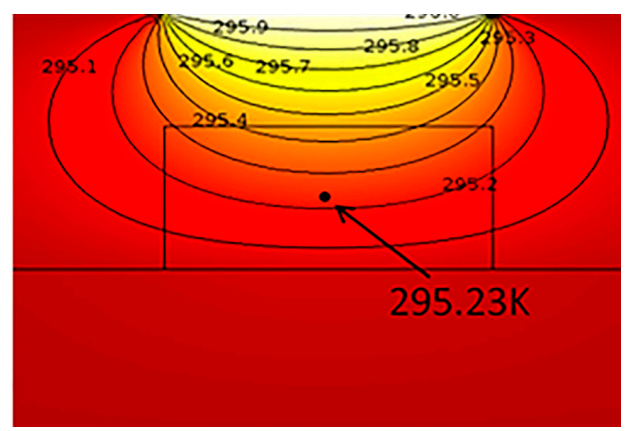

(b)

Figure 2. (a) The optical field of the fundamental mode in hybrid waveguide at $532 \mathrm{~nm}(\mathbf{b})$ the thermal field of the thermal optical switch.

We fabricated the device by the wet etching method. Figure 3 shows the device fabrication process. A $2.5 \mu \mathrm{m} \mathrm{SiO} 2$ layer was deposited on the silicon substrate as the under-cladding by the chemical vapor deposition (CVD) technique. A $3 \mu \mathrm{m}$ thickness SU-8-2005 was fabricated on the $\mathrm{SiO}_{2}$ layer by spin-coater (WS-650MZ-23NPPB). After baking, ultraviolet (UV) photo-etching (ABM/6/350) using a waveguide mask was performed for $4 \mathrm{~s}\left(20 \mathrm{~mW} / \mathrm{cm}^{2}\right)$, and then the post bake and developing was performed. A $4 \mu \mathrm{m}$ PMMA upper cladding layer was spun-coated and baked at $150{ }^{\circ} \mathrm{C}$ for $10 \mathrm{~min}$ with hot plate. Next, a $100 \mathrm{~nm}$ thick layer of aluminum was vaporized on the surface of the PMMA whileusing a coating machine (DM-300B), and the aluminum electrode was obtained by the BP218 mask wet etching method.

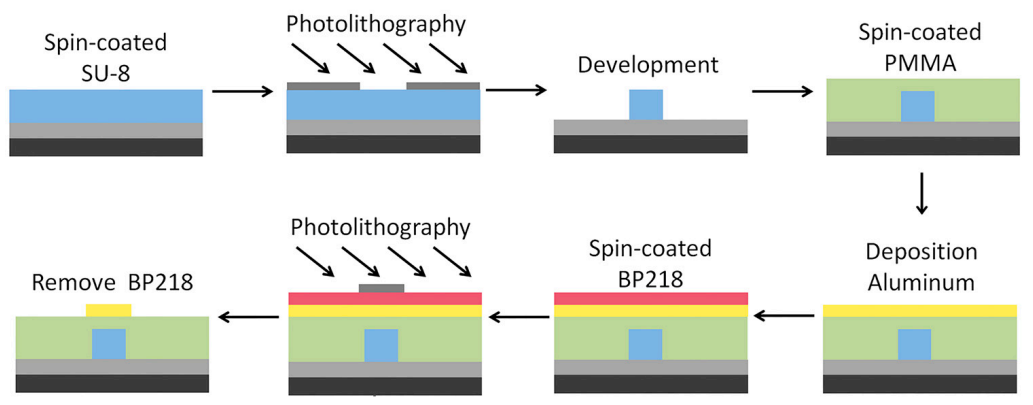

Figure 3. Process flow for fabricating the thermal optical switch. 
The prepared device was tested at the 532nm wavelength. Figure 4a shows the photograph of the test system. Figure $4 \mathrm{~b}$ shows the measured result. The rise time and fall time are $230 \mu \mathrm{s}$ and $280 \mu \mathrm{s}$. The extinction ratio is $4.8 \mathrm{~dB}$ and the corresponding voltage change value is $2.4 \mathrm{~V}$. The power consumption is $6.55 \mathrm{~mW}$, according to the resistance of the heating electrode $(0.88 \mathrm{k} \Omega)$.

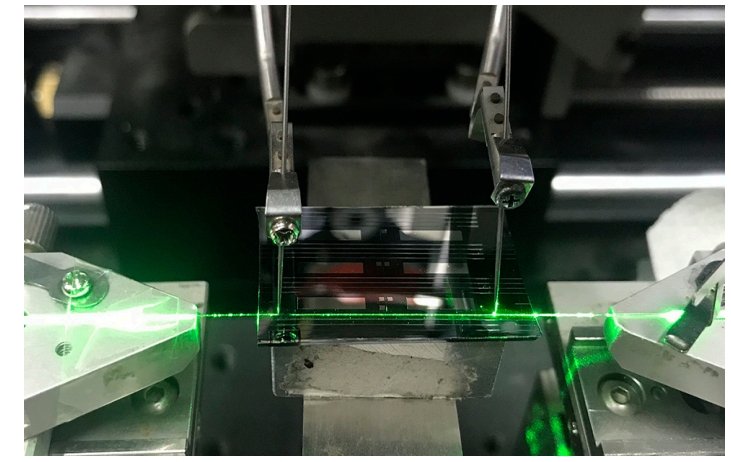

(a)

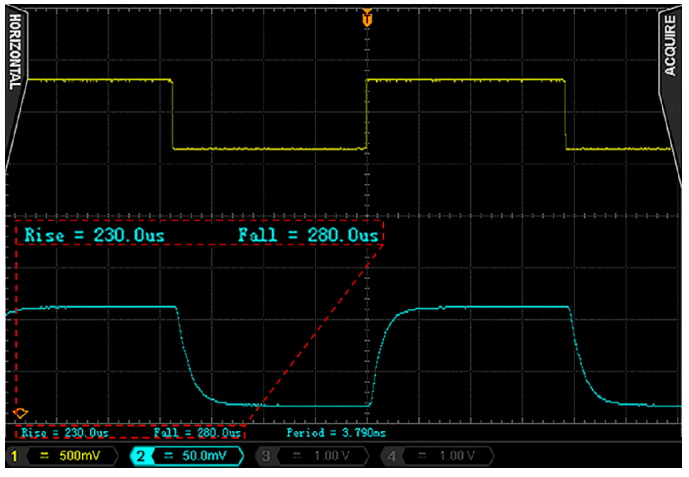

(b)

Figure 4. (a) The photograph of the device and its test system; and, (b) Switching response of the thermal optical switch.

The results indicate that the theoretical simulations accord with the test results. The switching time is faster than all polymer switches due to the high thermal conductivity of $\mathrm{SiO}_{2}$ lower cladding and silicon substrate. In addition, the device has lower power consumption than other polymer thermo-optic switches with the same structure due to its short wavelength. However, the extinction ratio of the device is relatively low. For the following discussion, the key issue is to improve the extinction ratio. It becomes a question of reducing the modes of the device.

\subsection{UV Writing EPO/Graphene Thermal Conduction Layer and Side Electrode}

UV-writing method is introduced to reduce the modes in the waveguide and increase the waveguide dimension. Figure 5 shows the UV-writing device structure. M-Z structure parameters are the same with the above wet etching thermal optical switch. EPO (micro resist technology GmbH, Berlin, Germany) is selected as the polymer waveguide core material due to its high stability and low optical loss. The exposure of EPO leads to the cross link of the material. The refractive index difference between the cross linked EPO and the uncross linked EPO is calculated to be 0.001 by the ellipsometer (SPEL M-2000VI). The refractive index of exposed EPO and unexposed EPO are 1.6099 and 1.6109, respectively. The thermal conductivity is $0.2 \mathrm{~W} /(\mathrm{m} \cdot \mathrm{K})$. The side electrode is introduced to reduce the switching time. The electrode is designed to fabricate adjacent to the waveguide due to the low absorption of the metal at $532 \mathrm{~nm}$. Two-dimensional (2D) material (such as graphene) layer is aimed to increase the heating efficiency. The graphene layer is designed to reduce the power consumption and switching time. The thermal conductor of the material is simulated with the parameter of $5300 \mathrm{~W} /(\mathrm{m} \cdot \mathrm{K})$.

Matlab calculates the relationship between the thickness and effective refractive index, as shown in Figure 6a. The exceptional loss that is caused by the side electrode is simulated in Figure 6b. With the electrode position at $1 \mu \mathrm{m}$ beside the waveguide, the absorption loss is $0.014 \mathrm{~dB} / \mathrm{cm}$. Figure $6 \mathrm{c}$ shows the thermal field with optimized side electrode and graphene thermal conduction layer. The UV-writing device is also simulated with the power consumption of $3.14 \mathrm{~mW}$ (without graphene) and $1.5 \mathrm{~mW}$ (with graphene). The side electrode UV-writing switching properties are calculated to be $52 \mu \mathrm{s}$ (rise) and $89 \mu$ s (down). The switch with assisted graphene has a switching time of $18.2 \mu \mathrm{s}$ (rise) and $85 \mu \mathrm{s}$ (down), as shown in Figure 6d. 


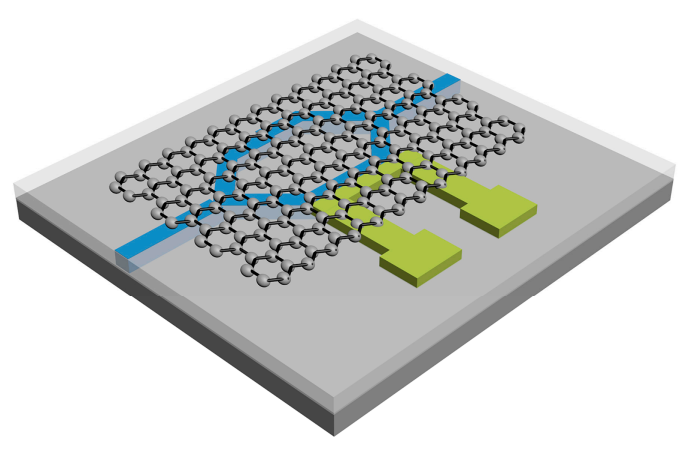

(a)

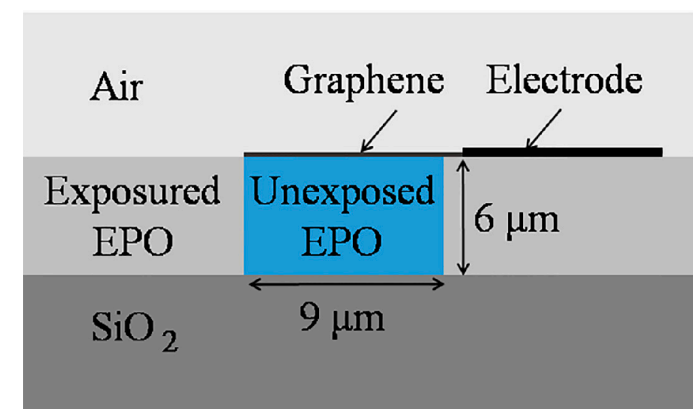

(b)

Figure 5. (a) The schematic of UV-writing thermal optical switch; (b) Schematic diagram of the cross-section of the device.

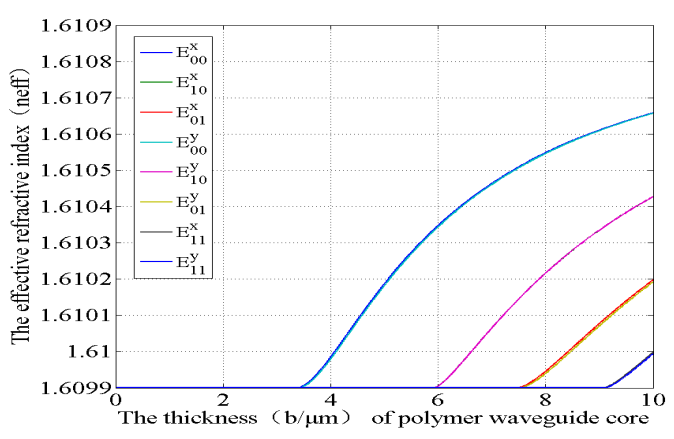

(a)

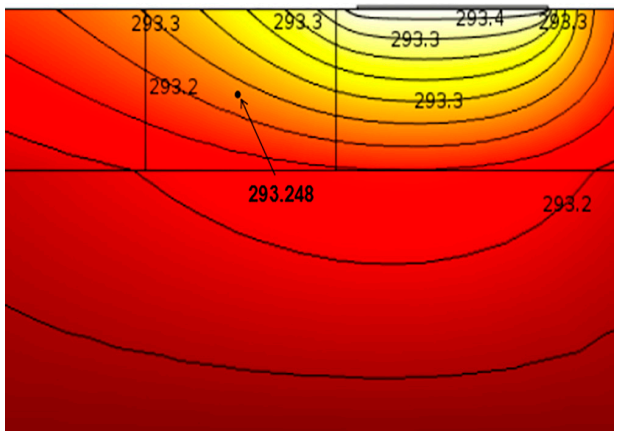

(c)

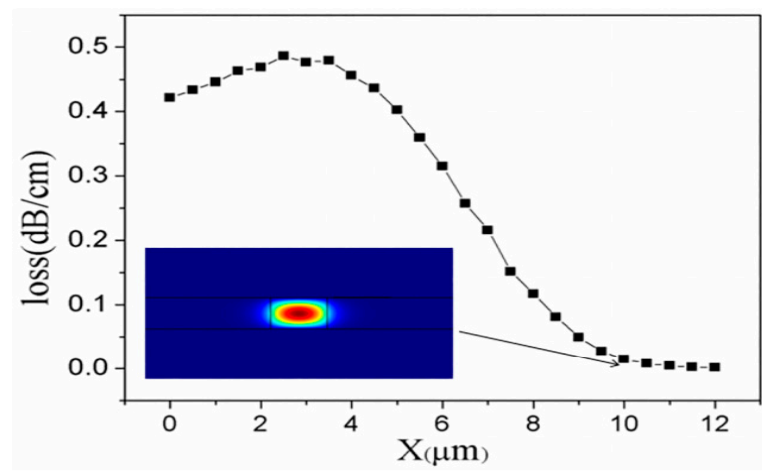

(b)

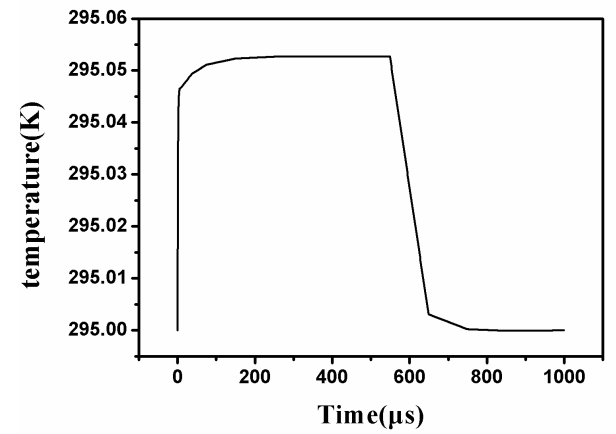

(d)

Figure 6. (a)The single mode condition of UV-writing waveguide; (b) The optical field and the absorption of the electrode. (c) The thermal field with side electrode and graphene conduction layer; and, (d) The switching properties of the UV-writing polymer optical switch.

We compare the results of the thermal optical witches in this paper with those of other reported polymer thermal optical switches [20,21], as shown in Table 1 . In Table 1, we can see that the TO switch at $532 \mathrm{~nm}$ would have lower power consumption, due to its short wavelength. Although the extinction ratio in this paper is lower, the low extinction ratio problem would be solved by the UV-writing technique. In addition, graphene has a relatively large thermal conductivity, which is conducive to reducing the switching response time and power consumption. 
Table 1. Comparisons among the performances of this thermal optical switch and those of other reported polymer thermal optical switches.

\begin{tabular}{|c|c|c|c|c|c|c|}
\hline Reference & $\begin{array}{c}\text { Structure } \\
\text { (Core/Upper-Clading/Under-Cladding) }\end{array}$ & $\begin{array}{l}\text { Wavelength } \\
\text { (nm) }\end{array}$ & ER (dB) & PC (mW) & RT ( $\mu \mathrm{s})$ & FT $(\mu s)$ \\
\hline 9 & ZPUcore/ZPUcladding/ZPUcladding & 1550 & $<-70$ & 200 & \multicolumn{2}{|c|}{$<10000$} \\
\hline 10 & $\mathrm{SiO}_{2}-\mathrm{TiO}_{2}-\mathrm{PMMA} / \mathrm{SiO}_{2}-\mathrm{PMMA} / \mathrm{SiO}_{2}-\mathrm{PMMA}$ & 650 & 8.2 & 22.6 & 400 & 780 \\
\hline 11 & SU-8/PMMA/SiO ${ }_{2}$ & 1550 & 18.3 & 13 & 73.5 & 96.5 \\
\hline 20 & Doped PMMA/PMMA/PMMA & 650 & 23.4 & 5.3 & 464.4 & 448.0 \\
\hline 21 & SU-8/PMMA/SiO 2 & 1550 & 26.5 & 7.2 & 106 & 93 \\
\hline This work & SU-8/PMMA/SiO & 532 & 4.8 & 6.55 & 230 & 280 \\
\hline $\begin{array}{l}\text { UV-writing with } \\
\text { graphene }\end{array}$ & $\mathrm{EPO} / \mathrm{air} / \mathrm{SiO}_{2}$ & 532 & - & 1.5 & 18.2 & 85 \\
\hline
\end{tabular}

${ }^{1} \mathrm{IL}$, insertion loss; ER, extinction ratio; PC, power consumption; RT, rise time; FT, fall time.

\section{Conclusions}

In this letter, a polymer-silica hybrid thermal optical switch at $532 \mathrm{~nm}$ was simulated and fabricated with low power consumption of $6.55 \mathrm{~mW}$ and the switching time of $0.23 \mathrm{~ms}$ (rise) and $0.28 \mathrm{~ms}$ (down). A UV-writing thermal optical switch with graphene layer was proposed in order to solve the low extinction ratio problem. The proposed optimized device has faster switching time (18.2 $\mu \mathrm{s}$ and $85 \mu \mathrm{s})$ and higher heating efficiency (power consumption of $1.5 \mathrm{~mW}$ ). Future work will be working on the fabrication of 2D material based UV-writing thermal optical device. The device can be used in the manipulation of the visible light communication and the wearable device.

Author Contributions: Conceptualization, Y.Y.(Yunji Yi); Data curation, J.L. and Y.C.; Formal analysis, J.L.; Funding acquisition, Y.Y.(Yunji Yi); Methodology, B.L.; Project administration, D.Z.; Resources, F.W.; Writing-original draft, Y.Y.(Yunji Yi); Writing-review \& editing, Y.S., Y.Y.(Yue Yang), and S.L.

Funding: This research was funded by National Natural Science Foundation of China $(61605057,61575076)$, Natural Science Foundation of Jilin Province (20190201190JC) and Excellent Youth Foundation of Jilin Province (20170520158JH).

Conflicts of Interest: The authors declare no conflict of interest.

\section{References}

1. Espinola, R.L.; Tsai, M.C.; Yardley, J.T.; Osgood, R.M. Fast and low-power thermooptic switch on thin silicon-on-insulator. IEEE Photonics Technol. Lett. 2003, 15, 1366-1368. [CrossRef]

2. Tsai, C.M.; Taga, H.; Yang, C.H.; Lo, Y.L.; Liang, T.C. Demonstration of a ROADM using cyclic AWGs. J. Lightw. Technol. 2011, 29, 2780-2784. [CrossRef]

3. Liang, L.; Zheng, C.T.; Sun, X.Q.; Wang, F.; Ma, C.S.; Zhang, D.M. Driving-noise-tolerant broadband polymer/silica hybrid thermo-optic switch with low power consumption. Fiber Integr. Opt. 2012, 31, 299-315. [CrossRef]

4. Liu, Y.F.; Wang, X.B.; Sun, J.W.; Sun, J.; Wang, F.; Chen, C.M.; Sun, X.Q.; Cui, Z.C.; Zhang, D.M. Improved performance of thermal-optic switch using polymer/silica hybrid and air trench waveguide structures. Opt. Lett. 2015, 40, 1888-1891. [CrossRef]

5. Zhang, Z.; Keil, N. Thermo-optic devices on polymer platform. Opt. Commun. 2016, 362, 101-114. [CrossRef]

6. Reilly, M.A.; Coleman, B.; Pun, E.Y.B.; Penty, R.V.; White, I.H.; Ramon, M.; Xia, R.; Bradley, D.D.C. Optical gain at $650 \mathrm{~nm}$ from a polymer waveguide with dye-doped cladding. Appl. Phys. Lett. 2005, 87, 231116. [CrossRef]

7. Rumbaugh, L.K.; Bollt, E.M.; Jemison, W.D.; Li, Y. A $532 \mathrm{~nm}$ chaotic lidar transmitter for high resolution underwater ranging and imaging. In Proceedings of the 2013 OCEANS-San Diego, San Diego, CA, USA, 23-27 September 2013; pp. 1-6.

8. Dooley, E.E.; Golaszewski, N.M.; Bartholomew, J.B. Estimating accuracy at exercise intensities: A comparative study of self-monitoring heart rate and physical activity wearable devices. JMIR mHealth uHealth 2017, 5, e34. [CrossRef]

9. Noh, Y.O.; Lee, H.J.; Won, Y.H.; Oh, M.C. Polymer waveguide thermo-optic switches with $-70 \mathrm{~dB}$ optical crosstalk. Opt. Commun. 2006, 258, 18-22. [CrossRef] 
10. Chen, C.M.; Han, C.; Wang, L.; Zhang, H.X.; Sun, X.Q.; Wang, F.; Zhang, D.M. 650 nm all-polymer Thermo-optic waveguide switch arrays based on novel organic-inorganic grafting PMMA materials. IEEE J. Quantum Electron. 2013, 49, 61-66. [CrossRef]

11. Liang, L.; Zheng, C.T.; Yan, Y.F.; Sun, X.Q.; Wang, F.; Ma, C.S.; Zhang, D.M. Low-power and high-speed thermo-optic switch using hybrid silica/polymer waveguide structure: Design, fabrication and measurement. J. Mod. Opt. 2012, 59, 1084-1091. [CrossRef]

12. Xie, N.; Hashimoto, T.; Utaka, K. Design and performance of low-power, high-speed, polarizationindependent and wideband polymer buried-channel waveguide thermo-optic switches. J. Lightw. Technol. 2014, 32, 3067-3073. [CrossRef]

13. Yu, Y.Y.; Sun, X.Q.; Ji, L.T.; He, G.B.; Wang, X.B.; Yi, Y.J.; Chen, C.M.; Wang, F.; Zhang, D.M. The 650-nm variable optical attenuator based on polymer/silica hybrid waveguide. Chin. Phys. B 2016, 25, 054101. [CrossRef]

14. Sun, D. Manipulation of the coherent spatial and angular shifts of Goos-Hänchen effect to realize the digital optical switch in silicon-on-insulator waveguide corner. J. Appl. Phys. 2016, 120, 183101. [CrossRef]

15. Satija, U.; Trivedi, N.; Biswal, G.; Ramkumar, B. Specific Emitter Identification Based on Variational Mode Decomposition and Spectral Features in Single Hop and Relaying Scenarios. IEEE Trans. Inf. Forensics Secur. 2019, 14, 581-591. [CrossRef]

16. Morozov, Y.; Lapchuk, A.; Le, Z.; Dong, Y.F. Efficient Background-Noise-Reduced Single-Mode Waveguides Platform for On-Chip Integrated Photonics. IEEE Photonics J. 2019, 11, 1-18. [CrossRef]

17. Wang, X.B.; Sun, J.; Chen, C.M.; Sun, X.Q.; Wang, F.; Zhang, D.M. Thermal UV treatment on SU-8 polymer for integrated optics. Opt. Mater. Express 2014, 4, 509-517. [CrossRef]

18. Ji, L.; He, G.; Gao, Y.; Xu, Y.; Liang, H.; Wang, X.; Yi, Y.; Chen, C.; Wang, F.; Sun, X.; et al. High sensitivity waveguide micro-displacement sensor based on intermodal interference. J. Opt. UK 2017, 19, 115804. [CrossRef]

19. Wang, X.B.; Sun, J.W.; Sun, J.; Sun, X.Q.; Chen, C.M.; Wang, F.; Zhang, D.M. Performance enhancement of strip-loaded electro-optic modulator using photobleaching-assisted method. Appl. Phys. B 2015, 118, 579-585. [CrossRef]

20. Wang, X.B.; Sun, J.; Liu, Y.F.; Sun, J.W.; Chen, C.M.; Sun, X.Q.; Wang, F.; Zhang, D.M. 650-nm $1 \times 2$ polymeric thermo-optic switch with low power consumption. Opt. Express 2014, 22, 11119-11128. [CrossRef]

21. Yan, Y.F.; Zheng, C.T.; Liang, L.; Meng, J.; Sun, X.Q.; Wang, F.; Zhang, D.M. Response-time improvement of a $2 \times 2$ thermo-optic switch with polymer/silica hybrid waveguide. Opt. Commun. 2012, 285, 3758-3762. [CrossRef]

(C) 2019 by the authors. Licensee MDPI, Basel, Switzerland. This article is an open access article distributed under the terms and conditions of the Creative Commons Attribution (CC BY) license (http://creativecommons.org/licenses/by/4.0/). 